Dieses Dokument ist eine Zweitveröffentlichung (Postprint) /

This is a self-archiving document (postprint):

Max Leuthäuser, Uwe Aßmann

Enabling View-based Programming with SCROLL: Using roles and dynamic dispatch for etablishing view-based programming

Erstveröffentlichung in / First published in:

MORSE/VAO '15: Proceedings of the 2015 Joint MORSE/VAO Workshop on Model-Driven Robot Software Engineering and View-based Software-Engineering, L'Aquila, 2015. ACM Digital Library, S. 25-33. ISBN 978-1-4503-3614-7

DOI: https://doi.org/10.1145/2802059.2802062

Diese Version ist verfügbar / This version is available on:

https://nbn-resolving.org/urn:nbn:de:bsz:14-qucosa2-707720 


\section{Enabling View-based Programming with SCROLL}

\section{Using roles and dynamic dispatch for etablishing view-based programming}

\author{
Max Leuthäuser \\ Software Technology Group \\ TU Dresden, Germany \\ max.leuthaeuser@tu-dresden.de
}

\author{
Uwe Aßmann \\ Software Technology Group \\ TU Dresden, Germany \\ uwe.assmann@tu-dresden.de
}

\begin{abstract}
Present-day software systems have to fulfill an increasing number of requirements rendering them more and more complex. Many systems need to anticipate changing contexts (self-adaptive systems) or need to adapt to changing business rules or requirements (self-optimizing systems). The challenge of 21th century software development will be to cope with these aspects. We believe that the role concept offers a simple way to adapt object-oriented programs to their changing contexts. In a role-based language, an object plays multiple roles during its lifetime. If the contexts are represented as first-class entities, they provide dynamic views to the object-oriented program, and if the context changes, the dynamic views can be switched easily, and the software system adapts automatically.

However, the concepts of roles and dynamic contexts have been discussed for a long time in many areas of computer science. So far, their implementation in an existing objectoriented language requires a specific runtime environment. Also, classical object-oriented languages and their runtime systems are not able to cope with essential role-specific features, such as true delegation or dynamic binding of roles. As a solution, this work presents a simple implementation pattern for role-based objects that does not require a specific runtime system, SCROLL (SCala ROles Language). The implementation pattern is demonstrated on the basis of the Scala language. As technical support from Scala, the pattern requires dynamic mixins, compiler-translated function calls, and implicit conversions. The implementation details of the pattern are hidden in a Scala library and therefore transparent to Scala programmers. The SCROLL library supports roles embedded in structured contexts, so-called compartments. We show that they are specific, hierarchic runtime views, which enables hierarchic view-based programming for free in Scala.

We also discuss how to apply the implementation pattern of SCROLL for other languages, in particular for behavioral modeling languages in MDSD. This discussion shows that
\end{abstract}

Permission to make digital or hard copies of all or part of this work for personal or classroom use is granted without fee provided that copies are not made or distributed for profit or commercial advantage and that copies bear this notice and the full citation on the first page. To copy otherwise, to republish, to post on servers or to redistribute to lists, requires prior specific permission and/or a fee.

VAO 2015, L'Aquila, Italy

Copyright 20XX ACM X-XXXXX-XX-X/XX/XX ...\$15.00. the SCROLL pattern can be embedded into the generated code, so that it still is hidden to the developer, but does not require a specific runtime system. Using the pattern in model-driven code generation enables dynamic views for all kinds of modeling languages. And therefore, this paper shows a way how to realize dynamic views for all modeling languages in MDSD.

\section{INTRODUCTION}

Let me try to explain to you, what to my taste is characteristic for all intelligent thinking. It is, that one is willing to study in depth an aspect of one's subject matter in isolation for the sake of its own consistency, all the time knowing that one is occupying oneself only with one of the aspects. We know that a program must be correct and we can study it from that viewpoint only; we also know that it should be efficient and we can study its efficiency on another day, so to speak. In another mood we may ask ourselves whether, and if so: why, the program is desirable. But nothing is gained - on the contrary! - by tackling these various aspects simultaneously. It is what I sometimes have called "the separation of concerns", which, even if not perfectly possible, is yet the only available technique for effective ordering of one's thoughts, that I know of. This is what I mean by "focusing one's attention upon some aspect": it does not mean ignoring the other aspects, it is just doing justice to the fact that from this aspect's point of view, the other is irrelevant. It is being one- and multiple-track minded simultaneously.

Edsgar W. Dijkstra, EWD 447,

http://www.cs.utexas.edu/users/EWD/ewd04xx/EWD447.PDF

Modeling dynamic and complex domains has been investigated for more than 35 years, starting with Charles W. Bachmann in 1977. He proposed role-based modeling 3] to capture both context-dependent and collaborative behavior of objects. Since then, many approaches in different research areas, ranging from data modeling [3, 19,32 , via conceptual modeling [42, 18] through to programming languages [5, 23 . 7. 37 emerged. The separateness of these research areas often leads to ignorance of the results of other fields. Consequently, the long period of research on role-based modeling had almost no influence on common software development practice. But as current software systems are characterized by increasing complexity and context-dependence 35], there is a strong demand for new concepts beyond the classical object-oriented design. In detail, while mainstream object-oriented modeling languages, e.g., the Unified Modeling Language (UML) 39, are good at capturing a systems structure, they lack ways to capture the systems behavior, as it dynamically emerges through collaborating objects 38. Roles are a natural concept capturing the behavior of participants in a collaboration. In addition to that, roles permit 
the specification of interactions independent from the interacting objects. Similarly, roles capture context-dependent behavior of objects, that is only exhibited when the role is played. In turn, the notion of roles can help to tame both increased complexity and context-dependence.

The functionality of complex software systems usually lies beyond the representative capabilities of a single type of software representation. Therefore, an increasingly large variety of heterogeneous representations (e.g. specifications, models, diagrams, programs etc.) are used in the various phases of software development to represent different aspects of a system's behavior and properties. These essentially represent different conceptual views of the software system, and usually present overlapping information that needs to be kept consistent. As Dijkstra said, the principle of separation of concerns $(\mathrm{SoC})$ requires that a complex problem or system should be looked at in views to simplify its engineering. Hence, techniques to form views, to manage them, and to re-integrate them with the base system are urgently looked for.

Traditional software engineering environments have always been based on a synthetic approach to views in which the different representations of a software system are treated as separate artifacts. As a consequence, the properties of the system under development have to be inferred by synthesizing the information spread over the different views, and the overall coherence of the information has to be ensured by maintaining a large number of pairwise "correspondences" between the separate artifacts. This principle of synthetic views underpinning traditional software engineering environments is also reflected in most of today's development methods. Indeed, software development by refinement, accompanied by pairwise traceability links to maintain information consistency, is essentially a synthetic one.

We believe it will be beneficial for software engineering, if projective approaches to the creation and evolution of views of software systems are adopted ("everything is a view"). In a projective software engineering environment, none of the views are stored permanently as separate artifacts. Instead, they are all derived on-the-fly from a single, central information source commonly called a single underlying model (SUM). This includes code-level views of the system: In a fully projective environment, there is no single special artifact called the code, which has a dominant role in the lifecycle of a project. Instead, code-level views of the system can be generated on the fly and can be used to add information, just like any other view. The SUM can then be optimized for executability, expressiveness etc. and is free of the need to be parsable. By definition, projective software engineering environments do not require the notion of correspondence links (e.g., traceability links) between views because all views are mutually consistent by virtue of the SUM. They also do not require a strict notion of linear refinement between views since they allow information to be added or changed at any time using the view type that is most appropriate for the stakeholder.

Also at runtime, the projection of systems and their runtime state into views is very important. Several researchers have suggested to establish a field called "models at runtime", in which the state of a self-adaptive system is maintained as one or several runtime models. In a projective models-at-runtime-system, every time a system enters a new context, a new context-specific view must be derived from the runtime $S U M$, the single underlying runtime model. During the lifetime of the context, the view must be connected to the system (the runtime SUM) in a causal connection, i.e., changes in the view must be re-integrated into the runtime SUM. When a system leaves the context, the view has to be disabled or abolished. Usually, many contexts, i.e., many views are active at the same time, so that views also influence each other and must be coordinated.

One of the fundamental concepts for view-based separation of concerns in object-oriented systems are roles. Though other forms of views are possible, roles extend the classical object model in a natural way. Roles can be related at runtime to a context as first-class object. (For instance, the rolebased language ObjectTeams 23 introduces Teams, simple runtime contexts for roles, as first-class language construct.) Then, the context forms a viewpoint, and its related set of roles a view of the software system. However, role-based separation of concerns at runtime level will fail at the moment. Many of the suggested role-based programming languages have been abandoned by their developers and do not provide a running compiler; others do not provide a runtime system compatible to one of the major platforms ${ }^{1}$ Therefore, we argue that it is necessary to establish the basic concepts of roles (views) and contexts (viewpoints) with an appropriate light-weight tooling, available in a major programming platform so that view-based programming becomes available for the masses. Such a light-weight approach is also beneficial to support different shades of the meaning of roles and contexts [31, because it can be adapted easily by an expert programmer or language engineer.

In this paper, we suggest an implementation pattern for roles and contexts based on dynamic mixins and compilertranslated function calls. The pattern can be hidden in a library, and we present the Scala library SCROLL as an example case study. The pattern can be realized in any language with these prerequisites, in particular in modeling languages with model-based code generation. The pattern uses the standard Java platform and runtime system, and offers dynamic views at runtime, to realize runtime SUM in standard Scala and Java application.

The remainder of this paper is structured as follows. First we summarize the properties of roles (Sec. 2) and views (Sec. 3) as additional introduction. We continue with a section presenting SCROLL (Sec. 4). We show how to use dynamic marker traits, compiler-translated function calls and implicit conversions to realize roles and hierarchic views in Scala. Finally, the evaluation (Sec. 5 tries to classify our work in the context of other contemporary approaches and discusses how to transfer it to modeling languages.

\section{PROPERTIES OF ROLES}

This section gives an introduction to the properties of roles by analyzing their features. Even if roles have been studied for a long time, the first thorough analysis of them was not published until the year 2000 by Friedrich Steimann 42 . He identified 15 features of roles that are useful to classify and compare all subsequent approaches. Since then, many languages utilizing roles have been published. However, only two applied Steimann's classification scheme, namely 11] and 24. In this work we will also use the listed features as introduction to the properties of roles, as well as evaluation

\footnotetext{
${ }^{1}$ ObjectTeams forms a notable exception in both points.
} 
1. Roles have properties and behaviors.

2. Roles depend on relationships.

3. Objects may play different roles simultaneously.

4. Objects may play the same role (type) several times.

5. Objects may acquire and abandon roles dynamically.

6. The sequence of role acquisition/removal may be restricted.

7. Unrelated objects can play the same role.

8. Roles can play roles.

9. Roles can be transferred between objects.

10. The state of an object can be role-specific.

11. Features of an object can be role-specific.

12. Roles restrict access.

13. Different roles may share structure and behavior.

14. An object and its roles share identity.

15. An object and its roles have different identities.

16. Relationships between roles can be constrained.

17. There may be constraints between relationships.

18. Roles can be grouped and constrained together.

19. Roles depend on compartments.

20. Compartments have properties and behaviors.

21. A role can be part of several compartments.

22. Compartments may play roles like objects.

23. Compartments may play roles which are part of themselves.

24. Compartments can contain other compartments.

25. Different compartments may share structure and behavior. 26. Compartments have their own identity.

Figure 1: Fiedrich Steimann's 15 classifying features (1-15), extracted from 42 and the additional ones (16-26) w.r.t. to the context-dependent nature of roles 31 .

criteria. On top of that, the following additional features of roles w.r.t. their context-dependent nature are extracted from 31. All features are enumerated more compactly in Fig. 1 .

16. Relationships between roles can be constrained. If roles depend on relationships, then it might be possible to further constrain them by intra-relationship constraints 19, 7, 34, i.e. irreflectivity, total order or exclusive parthood.

17. There may be constraints between relationships. In contrast to feature 16, this property suggests the existence of inter-relationship constraints, like the subset constraint 20 , $19,10,34$.

18. Roles can be grouped and constrained together. Most approaches suggesting to constrain roles 12, 11, 8, do not permit to group them and apply constraints to a whole group of related roles as suggested in 44,24 .

These three properties specify ways to constrain roles, but do not account for their context-dependence. The use of the term context leads to a dichotomy of its meaning. According to Dey 13, "context [represents] any information that can be used to characterize the situation of an entity". Thus, everything that can be attributed to an object in a situation contributes to its context. But within modeling languages, context represents a collaboration or container of a fixed, limited scope $15,27,38,30$. To overcome this dichotomy, researchers avoided the term context by using other terms, i.e. Environments 44], Institutions 4], Teams 23 and Ensembles 22. In turn, we use the term Compartment as a generalization of these terms to denote an objectified collaboration with a limited number of participating roles and a fixed scope.

19. Roles depend on compartments. Roles are dependent on some sort of context. We call them compartments $23,44,4$, 15. 27, 38, 30, 22. A typical example of a compartment is a university, which contains the roles Student and Teacher collaborating in Courses 24, 6, 33.

20. Compartments have properties and behaviors like objects $15,27,38,30$.

21. A Role can be part of several compartments 4, 44 15, 32. This property suggests that a role can be part of more than one compartment. Consider again the role type Teacher. It can be used in different compartments, i.e., School or University, where it might be implemented and constrained differently 4 .

22. Compartments may play roles like objects. While most approaches use compartments as a grouping mechanism, compartments can be seen as entities similar to naturals being able to play roles, as well 15,24 .

23. Compartments may play roles which are part of themselves. Continuing the argument of feature 22, compartments might be allowed to play roles belonging to the same compartments, as possible in 15, 24].

24. Compartments can contain other compartments. $24,27$. 29. This nesting is proposed to further structure compartments into smaller sub-compartments 24, 29 and, e.g. enables the representation of a university containing academic departments which in turn contain faculties.

25. Different compartments may share structure and behavior 26, 27. Compartments may inherit properties, features, roles, and constraints from each other. However, to fully support inheritance and polymorphism of compartments, the rules of family polymorphism have to be applied [26].

26. Compartments have their own identity. This feature is acknowledged by all approaches who treat compartments as first-class entities of the instance level [44, 24, 32, 38 29,22 . This feature is a prerequisite for the existence of compartments at runtime.

Researchers have successfully applied the concept of roles to the domain of context-aware systems. This has led to a number of new features attributed to roles affecting both model and instance level. Surprisingly, the definitional dependence of roles 17 is still applicable to compartments representing the definitional boundary and execution scope for their enclosing roles. Hence, the first 18 features highlight the relational nature of roles whereas the last eight emphasize the context-dependent nature of roles.

\section{PROPERTIES OF VIEWS}

A view is a representation of a whole system from the perspective of a related set of concerns. This meets Dijkstra's vision not to tackle all aspects of a program at once which would be highly ineffective. In this sense, Dijkstra's ideas also inspired the IEEE proposal for a standard for software architecture 28. where a set of related concerns is called viewpoints. They govern views (in the sense that each view conforms to exactly one viewpoint) and generate them by projecting parts of a system. For instance, the architectural viewpoint comprises several architectural concerns, such as coarse-grain structure, run-time process structure and run-time connections. This is distinguished from the application-specific viewpoint with the application-specific details of the implementation of components.

Therefore we assume in the following that views are partial and constructive representations of a system if they can be composed to the full representation of it. Several details are important when analyzing the features of views in the sense of modeling, programming and implementing the 
before-mentioned composability. We start with contemplating on the composition of views itself. View-based composition needs a merge (symmetric composition) and extend (asymmetric composition) operator. The first is commutative, merging views symmetric, while the latter (i.e. extension of components) can be considered to be asymmetric. Furthermore, both can be implemented in terms of each other. Hence, the construction (composition, merge) and projection (decomposition, split) are two sides of one coin. To continue, views can be defined as projective if they project the full representation of the system to something more simple. Projection extracts a view from the full representation of the system. Last but not least, views require open definitions (objects that can be re-defined, i.e. extended several times by different viewpoints).

\section{INTRODUCING SCROLL}

This section presents a light-weight implementation pattern for roles and context for dynamic view-based programming. The pattern is demonstrated with the SCROLL ${ }^{2}$ (SCala ROles Language) library, a small Scala package that allows for augmenting an object's type at runtime with dynamic role types embedded in reified contexts, so-called compartments. They are related to a set of roles. Entering such a context within a running system will activate its compartment and all its related roles (if they are bound to player objects). Hence, a compartment is related to a subset of a system (a view), i.e., all its related roles, which can be switched on and off by its activation or deactivation. When activating a compartment relating to a context, the roles of the compartment are merged into their players. Therefore, compartment-based composition switches on and off context-specific views, and compartments in SCROLL are viewpoints switching on and off dynamic views of the system. Additionally, SCROLL compartments may be nested. Therefore, compartments form hierarchical views, and switching on a compartment means to activate all views of all enclosing ones. To demonstrate that the concept of compartments can mimic the one of views the features of compartments are adapted and applied as shown in Sec. 5

The implementation pattern used in the SCROLL library requires two technical aspects of Scala. First, the dynamic marker trait. All calls to role functions, i.e., functions that are not natively available on the player object, are translated by the compiler using specific translation rules ${ }^{3}$ At runtime, the mapping of player objects to their roles is maintained with an acyclic directed graph (DAG), whose state influences the behavior of the dynamic role dispatch: If roles are deactivated, they are bypassed by the dispatch algorithm; if roles are activated, they take part in dispatch. Additionally, the definition of the actual dispatching rules can be influenced by the specification of functions for graph traversals on the object-player DAG. Because these functions can be composed, interesting dispatching schemes can be realized.

Consider the following example. As visualized in Fig. 2 , sometimes it is ambiguous, which role is responsible for answering a call to a given function (cmp. the call to function()). In SCROLL, the correct dispatch algorithm for the call is stored as dispatch function in an implicit variable, which can be passed implicitly to any function call on

$\sqrt[2]{\text { https://www.github.com/max-leuthaeuser/SCROLL }}$ 3 www.scala-lang.org/api/current/\#scala.Dynamic a role-playing object. A sketch of the Scala library code for role-based dynamic dispatch is shown in the following listing:

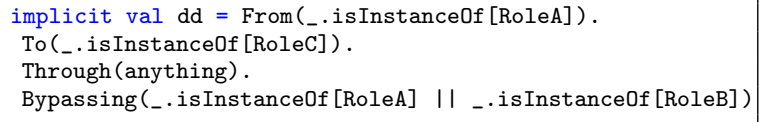

The correct dispatch behavior for the call is stored in variable dd. Due to the implicit keyword, it can be passed implicitly to any function call on a role-playing object, and this leads to the selection of the appropriate context-specific variant of the function function: When the function is called, along any path in the object-role-playing graph from RoleA to RoleC, (calls to From, Through, and To) the roles RoleA and RoleB will be skipped (call to Bypassing), before a role RoleC is found that is invoked. On the downside compilertranslated function calls cannot be debugged easily. Applying compiler rewrite rules hides important typing information to the tooling typically used by most developers, i.e. IDEs with debugger and link tracers. Writing plugins for those IDEs (e.g. Eclipse, Intellij) would overcome this issue and is currently under development.

Second, the implementation pattern in the SCROLL library relies on implicit conversions. Scala's implicit classes allow for packing player and role types into compound dynamic types. All important role features are exposed this way, e.g. adding, removing and transferring roles or accessing role functions and attributes. We illustrate this with a larger example (Fig. 3 and Fig. 4 ).

The implementation of a robot is separated into four views: one regarding its navigation (NavigationView), one for querying its sensors (SensorView), one for utilizing its actors (ActorView) and finally one for specifying certain behavior (BehaviorView). For the sake of simplicity, the views here only contains one role each, like the ServiceRole, which offers simple movement. In a real system, multiple implementations encapsulated in roles of different kinds of behavior can be considered. Accessing role-related features, like attributes or functions that are not natively available on the current instance of a player or role object (e.g., the attribute name in Line 6 of Fig. (4) are accessible through the +-Operator, which implicitly converts the current instance to a compound dynamic type enabling the application of the compiler rewrite rules w.r.t. the Dynamic Marker Trait as explained in Sec. 4. After specifying the plays-relationship between all roles and the player instance (Fig. 4 on line 30) the views can be merged. This provides symmetric view-based composition, whereas asymmetric composition (i.e., extension) is available through the standard inheritance mechanism from Scala itself.

Using implicit conversions, the compiler-translated function calls, the representation of roles and compartments can be completely hidden. Therefore, view decomposition of a runtime SUM as well as view composition, the merging of different views into the runtime SUM, is very simple using the SCROLL library.

\section{EVALUATION}

It is necessary to investigate how well the implementation with SCROLL using and merging compartments as views as well as binding roles dynamically blends into contemporary approaches. Thus, we use the previously defined classifica- 


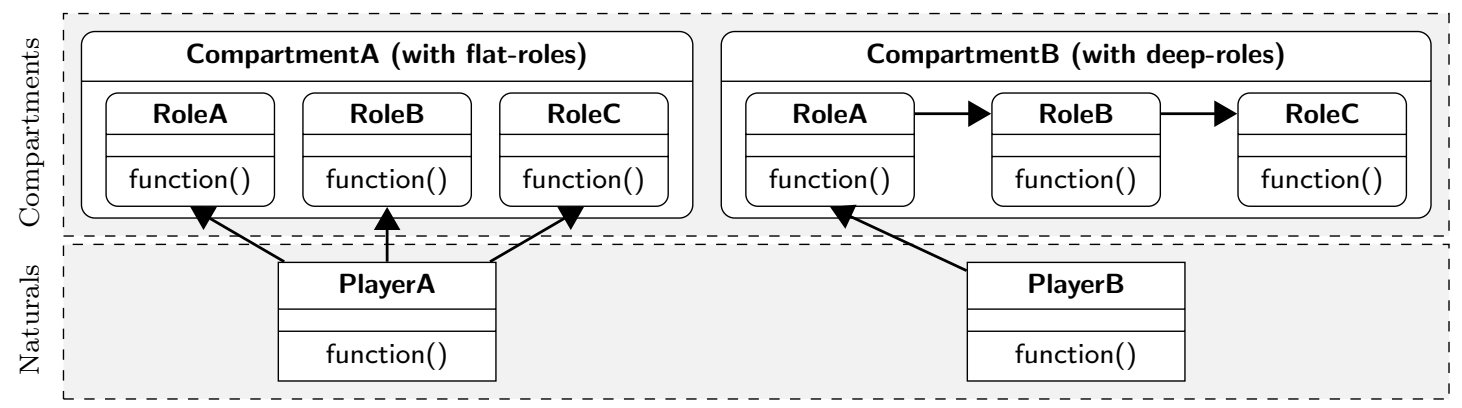

Figure 2: An example for the need of customizable role dispatch. It is ambiguous which role is responsible for answering a call to function(). Flat-roles (roles can not play roles themselves, left side) or deep-roles (right side) are semantically the same and are introducing the same ambiguity here.

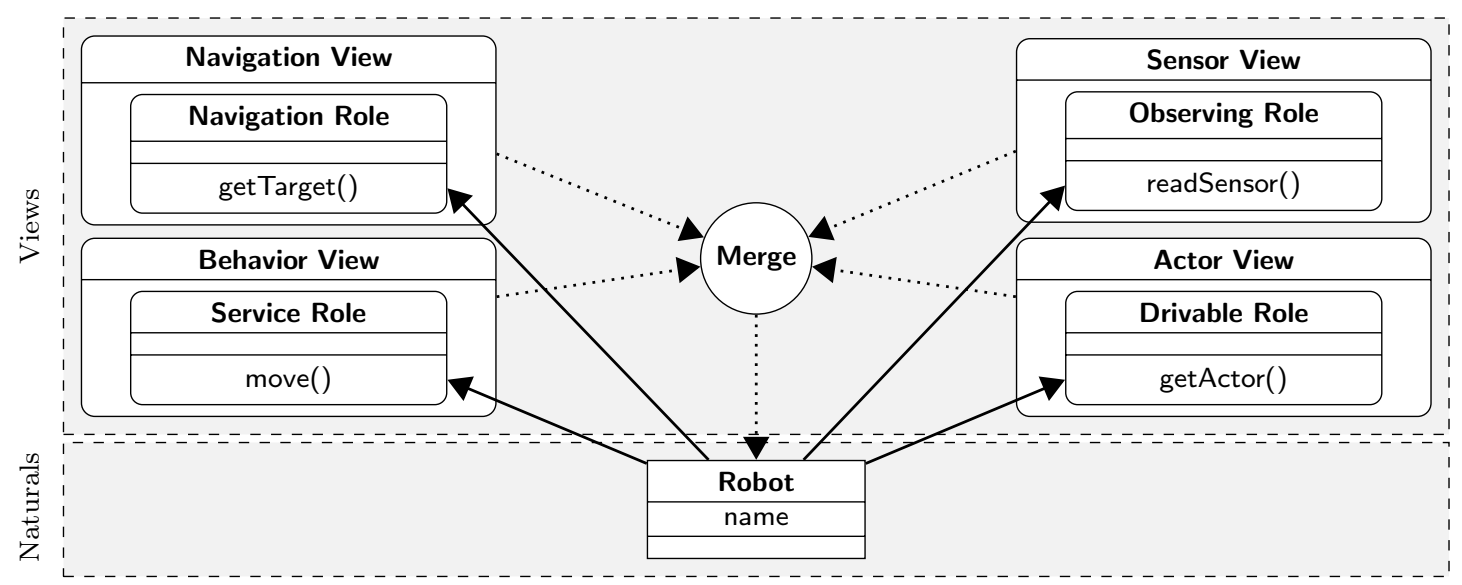

Figure 3: Class Robot is constructed (dotted arrows) from different views and plays (solid arrows) the contained roles.

tion scheme for checking the fulfillment of each feature and apply it to views instead of compartments. Features 1-15 are omitted here due to the fact that they are not related to the context-dependent nature of roles, i.e. to views/compartments.

16. Relationships between roles can be constrained. No. Since there are no first class relationships yet no constraints can be applied.

17. There may be constraints between relationships. No. Since there are no first class relationships yet no constraints can be applied.

18. Roles can be grouped and constrained together. Partly. They can be grouped into views, but role groups are not implemented yet, neither are constraints.

19. Roles depend on views. No. Technically one can import roles from anywhere. Implementing them directly in compartments might be beneficial (name spacing).

20. Views have properties and behaviors. Yes. Since compartments are implemented as classes (or case classes) they can have properties (as class attributes) and behavior (as arbitrary functions or methods).

21. A role can be part of several views. Partly. Technically one can import roles from anywhere so having them in multiple, nested views is no problem.

22. Views may play roles like objects. Yes. Any type is allowed to play roles at any time.

23. Views may play roles which are part of themselves. Yes. Since there are no restrictions concerning the type of the role a view might play.

24. Views can contain other views. Yes. Hierarchically nested views are supported.

25. Different views may share structure and behavior. Yes. Since views are standard Scala classes or case classes they support inheritance.

26. Views have their own identity. Yes. Since views are standard Scala classes or case classes they carry their own identity.

A compact overview is given in table 1. Most of the role features in question are supported. Using compartments as views and merging them in addition to the dynamic process of role binding within SCROLL enables view-based programming without the need of a custom compiler.

\section{RELATED WORK}

This section summarizes and compares how different runtime environments or technical spaces could be used to adapt view-based programming.

\subsection{Compartments with other languages}

First of all, SCROLL requires the concept of a dynamic marker trait, i.e., a dynamic mixin to an object. However, only few programming languages have incorporated this construct. In languages with static mixins (such as C\# 4.0), roles can be implemented by libraries, but they can be activated and deactivated only at allocation time of objects. This concept of static roles is similar to static mixins in mixin layers 41]. Therefore, for a realistic representation of dynamic compartment-based views, dynamic mixins are required from the base language. Nevertheless, if the concept is not available, it may be possible to employ a design 


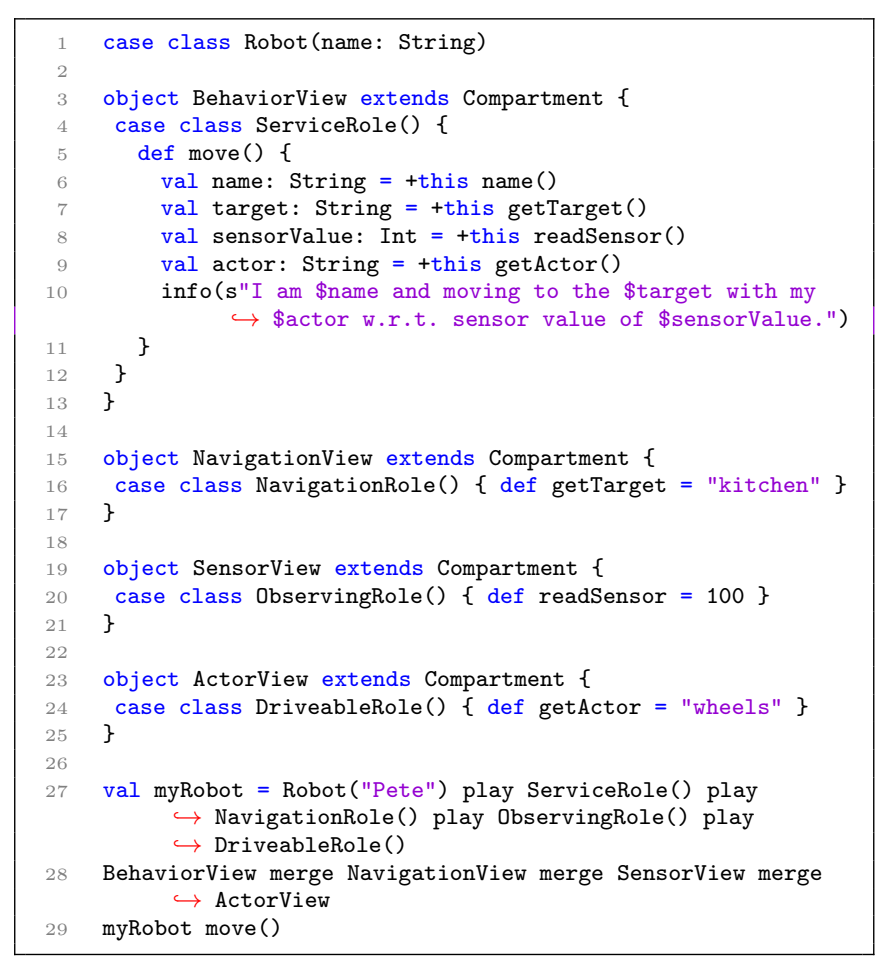

Figure 4: The robot is constructed from views.

pattern in the library, such as the Role-Object Pattern 9], to provide dynamic mixins simulated by decorator chains. Such a pattern can always then be added as a workaround, if the language meets the requirements. On the downside, the Role-Object Pattern does not directly support hierarchies of views and needs to be implemented carefully to avoid object schizophrenia [2].

If the language offers dynamic mixins, the concept of compartments, i.e., hierarchical views, can be implemented in a similar way as in this paper. In particular, dynamic mixins may also be available in modeling languages, at least with the help of code generation. In the final step of model-driven software development, when code is generated from the models, patterns such as Role-Object Pattern can be employed for simulated dynamic mixins of objects. This scheme is used in the SMAGS system for a flexible implementation of a role-based architectural language 36. On top of such a code generation scheme, a SCROLL-like library can easily be implemented. This indicates that the SCROLL approach is also very useful for modeling languages, because it requires a minimal set of features from the language, and nevertheless, provides hierarchical views. And finally, some modeling environments, such as the Eclipse Modeling Framework (EMF), already provide dynamic proxies, on which dynamic mixins can be built.

\subsection{Multiple Inheritance / Traits}

Although the concepts of multiple inheritance and traits are semantically perfectly fine to implement roles at runtime, they will lead to a very static system with an exponential blowup in the number of required classes for every new view or compartment one needs to add. Additionally, parallel object hierarchies may occur where cross-tree constraints are very hard to maintain.

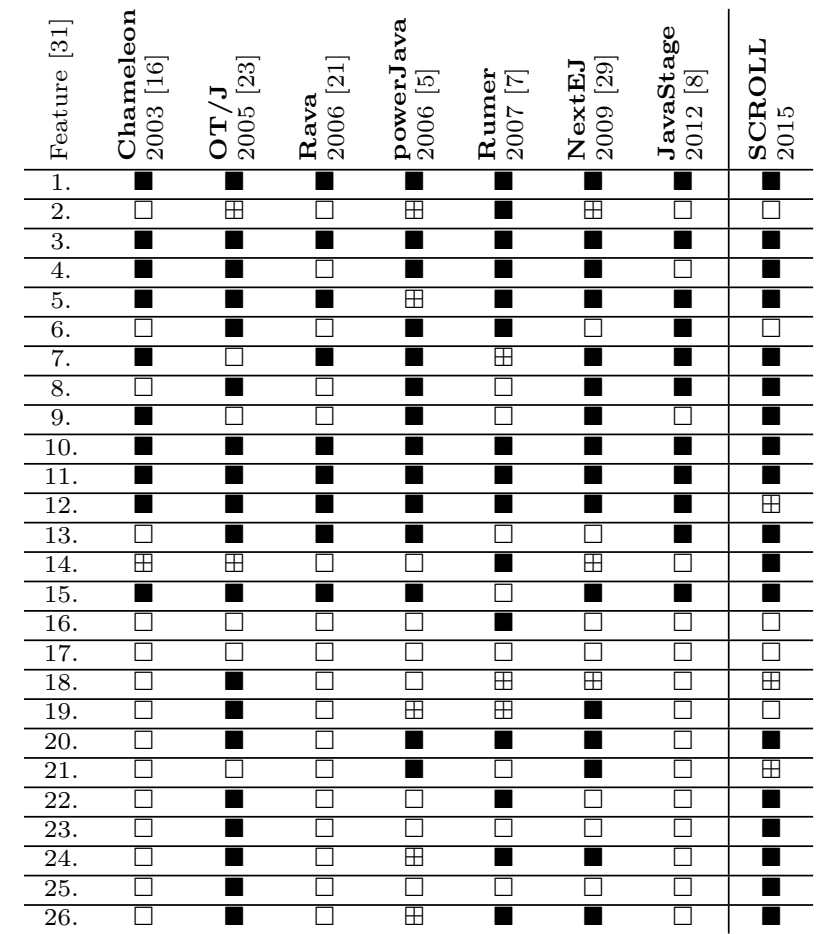

Table 1: Comparison of coeval approaches for etablishing roles at runtime based on 26 classifying features extracted from the literature 31, 42]. It differentiates between fully $(\square)$, partly $(\boxplus)$ and not supported $(\square)$ features.

\subsection{Aspect- / Subject-oriented programming}

Aspect-oriented programming allows to implement crosscutting concerns via joint-points and pointcuts. Often the composition is done statically although there exist a few dynamic approaches. E.g. ObjectTeams/Java (OT/J) 23] uses dynamic aspect weaving at bytecode-level for adding role-specific behavior to its players. Subject-oriented programming utilizes different class hierarchies from different perspectives which are comparable to view-based programming. On the downside there is no real composition language and the set of composition operators is fixed. Furthermore, no real control flow on the composition itself exists.

\subsection{Delegation and Delegation-Layers}

Basically delegation mimics the inheritance mechanism on object level. This requires (the generation of) a lot of management code and leads to object schizophrenia [2]. Delegation-Layers on the other hand define layers that group behavior for sets of objects and for sets of classes. Sadly, it implies fixed hierarchies and thus a system design that is too static.

\subsection{Other role-based programming languages}

Interestingly, most of the existing role-based programming languages are extensions to Java. They are either compiled to Java source code $16,21,5,8$ or to bytecode 23 directly. The first class of these languages focuses mainly on implementing objects playing roles.

Chameleon 16 features roles with so called constituent methods allowing to overwrite methods of their players, which work like advices in aspect-oriented programming. However, the major drawback of Chameleon is the fact that roles ex- 
tend their player to gain access to the player, which is both conceptually wrong [42] and limits the flexibility of roles. Rava 21] overcomes these issues by employing the RoleObject-Pattern 9] extended with the Mediator-Pattern [14]. They use special keywords to steer the generation of the necessary management code. Due to the use of the Role-Object Pattern and generation to plain Java, this solution suffers from object schizophrenia 25. JavaStage 8 eludes this problem, by only supporting static roles, i.e., the roles are directly compiled into the possible players as inner classes. To avoid name clashes, it employs a customizable method renaming strategy. Its main advantages are the capability to specify a list of required methods instead of a specific player class. Surprisingly, this approach limits itself to static roles unable to represent their relational and contextdependent nature. We proceed with Rumer [7], which contributes relationships as first class citizens and modular verification over shared state. Furthermore, it provides several intra-relationship constraints usable to restrict these relationships. Roles are the named places of a relationship with attributes and methods but without inheritance. Despite that, roles are only accessible within a relationship and not from their player. The most sophisticated approach to context-dependent roles so far is ObjectTeams/Java $(\mathrm{OT} / \mathrm{J})$ 23. Similar to Chameleon above, OT/J allows to override methods of their player by aspect weaving. Besides that, it introduces Teams to represent compartments whose inner classes automatically become roles. Notably, OT/J supports both the inheritance of roles and teams whereas the latter leads to family polymorphism 26. On the downside, it does neither support multiple unrelated player types for a role type nor first class relationships and only a limited form of constraints. This is similar to powerJava 1], which also introduces compartments, denoted Institutions, whose inner classes represent roles. However, powerJava features the distinction between role interface and role implementation where the former is callable from outside a specific institution and the latter is the institution-specific implementation of the same interface. Both Rava and powerJava are the only research prototypes providing a working compiler. Nevertheless, the project has been abandoned 43. A more recent approach towards context-oriented programming is NextEJ 29] as the successor of EpsilonJ [40]. It provides Contexts as first class citizens which do not only group roles but also represent an activation scope at runtime. These context activation scopes can be nested and act as a barrier where all roles are instantiated and bound automatically. So far, they only published their type-system of the core calculus and no compiler for NextEJ.

Consequently, all systems containing objectified contexts as first class citizens, e.g. Environments 44], Institutions 4], Teams 23] and Ensembles 22] like SCROLL does (i.e. with Compartments) are suitable for adaption w.r.t. establishing views at runtime. This has to be investigated in the future, up to our knowledge there is currently no literature available on this.

\section{CONCLUSIONS}

This paper presented an implementation pattern for rolebased objects and their hierarchical contexts in Scala, based on dynamic marker traits, compiler-translated function calls, and implicit conversions. The SCROLL library provides view-based programming on top of a standard Scala plat- form. SCROLL roles and compartments can help to handle both increased complexity and context-dependence of software systems, because with its light-weight approach, projective views on a runtime SUM are made available. The SCROLL approach can be transfered to other languages as well, in particular to modeling languages with model-based code generation. We believe that this is a contribution to the ubiquitous adoption of projective view-based approaches to software engineering - supporting the design metaphor that "everything is a view".

\section{Acknowledgments}

This work is funded by the German Research Foundation (DFG) within the Research Training Group "Role-based Software Infrastructures for continuous-context-sensitive Systems" (GRK 1907).

\section{REFERENCES}

[1] E. Arnaudo, M. Baldoni, G. Boella, V. Genovese, and R. Grenna. An implementation of roles as affordances: powerJava, Aug. 312009.

[2] U. Aßmann. Invasive Software Composition. Springer-Verlag, 2003.

[3] C. W. Bachman and M. Daya. The role concept in data models. In Proceedings of the Third International Conference on Very Large Data Bases, pages 464-476, Tokyo, Japan, 6-8 Oct. 1977.

[4] M. Baldoni, G. Boella, and L. Van Der Torre. powerjava: ontologically founded roles in object oriented programming languages. In Proceedings of the 2006 ACM symposium on Applied computing, pages 1414-1418. ACM, 2006.

[5] M. Baldoni, G. Boella, and L. van der Torre. Roles as a coordination construct: Introducing powerjava. Electr. Notes Theor. Comput. Sci, 150(1):9-29, 2006.

[6] S. Balzer, P. Eugster, and T. Gross. Relations: Abstracting object collaborations, Feb. 062008.

[7] S. Balzer, T. Gross, and P. Eugster. A relational model of object collaborations and its use in reasoning about relationships. In E. Ernst, editor, ECOOP, volume 4609 of Lecture Notes in Computer Science, pages 323-346. Springer, 2007.

[8] F. S. Barbosa and A. Aguiar. Modeling and programming with roles: introducing javastage. Technical report, Instituto Politécnico de Castelo Branco, 2012.

[9] D. Bäumer, D. Riehle, W. Siberski, and M. Wulf. The role object pattern. In Washington University Dept. of Computer Science, 1997.

[10] G. Bierman and A. Wren. First-class relationships in an object-oriented language. In ECOOP 2005 Object-Oriented Programming, pages 262-286. Springer-Verlag, 2005.

[11] G. Boella and L. Van Der Torre. The ontological properties of social roles in multi-agent systems: Definitional dependence, powers and roles playing roles. Artificial Intelligence and Law, 15(3):201-221, 2007.

[12] M. Dahchour, A. Pirotte, and E. Zimányi. A generic role model for dynamic objects. In Advanced Information Systems Engineering, pages 643-658. Springer, 2002. 
[13] A. K. Dey. Understanding and using context. Personal and ubiquitous computing, 5(1):4-7, 2001.

[14] E. Gamma, R. Helm, R. Johnson, and J. Vlissides. Design patterns: elements of reusable object-oriented software. Pearson Education, 1994.

[15] V. Genovese. A meta-model for roles: Introducing sessions. Roles' 07, page 27, 2007.

[16] K. B. Graversen and K. Østerbye. Implementation of a role language for object-specific dynamic separation of concerns. In AOSD03 Workshop on Software-engineering Properties of Languages for Aspect Technologies, 2003.

[17] N. Guarino and C. A. Welty. An overview of ontoclean. In Handbook on ontologies, pages 201-220. Springer, 2009.

[18] G. Guizzardi and G. Wagner. Conceptual simulation modeling with onto-uml. In Proceedings of the Winter Simulation Conference, page 5. Winter Simulation Conference, 2012.

[19] T. Halpin. ORM 2. In On the Move to Meaningful Internet Systems 2005: OTM 2005 Workshops, pages 676-687. Springer, 2005.

[20] T. A. Halpin. Object-role modeling (orm/niam). In Handbook on Architectures of Information Systems, pages 81-102. Springer, 1998.

[21] C. He, Z. Nie, B. Li, L. Cao, and K. He. Rava: Designing a java extension with dynamic object roles. In Engineering of Computer Based Systems, 2006. ECBS 2006. 13th Annual IEEE International Symposium and Workshop on, pages 7-pp. IEEE, 2006.

[22] R. Hennicker and A. Klarl. Foundations for ensemble modeling-the Helena approach. In Specification, Algebra, and Software, pages 359-381. Springer, 2014.

[23] S. Herrmann. Programming with roles in ObjectTeams/Java. Technical report, AAAI Fall Symposium, 2005.

[24] S. Herrmann. A precise model for contextual roles: The programming language objectteams/java. Applied Ontology, 2(2):181-207, 2007.

[25] S. Herrmann. Demystifying object schizophrenia. In Proceedings of the 4 th Workshop on MechAnisms for SPEcialization, Generalization and inHerItance, MASPEGHI '10, pages 2:1-2:5, New York, NY, USA, 2010. ACM.

[26] S. Herrmann, C. Hundt, and K. Mehner. Translation polymorphism in object teams. Technical report, TU Berlin, 2004.

[27] J. Hu and M. Liu. Modeling context-dependent information. In Proceedings of the 18th ACM conference on Information and knowledge management, pages 1669-1672. ACM, 2009.

[28] IEEE. Ieee 1471-2000 conceptual framework for architectural description. http://www.isoarchitecture.org/42010/cm/cm-1471-2000.html, 2000.

[29] T. Kamina and T. Tamai. Towards safe and flexible object adaptation. In International Workshop on Context-Oriented Programming, page 4. ACM, 2009.

[30] T. Kamina and T. Tamai. A smooth combination of role-based language and context activation. FOAL
2010 Proceedings, page 15, 2010.

[31] T. Kühn, M. Leuthäuser, S. Götz, C. Seidl, and U. Aßmann. A metamodel family for role-based modeling and programming languages. In

B. Combemale, D. Pearce, O. Barais, and J. Vinju, editors, Software Language Engineering, volume 8706 of Lecture Notes in Computer Science, pages 141-160. Springer International Publishing, 2014.

[32] M. Liu and J. Hu. Information networking model. In Conceptual Modeling-ER 2009, pages 131-144. Springer, 2009.

[33] M. Liu and J. Hu. Modeling complex relationships. In Database and Expert Systems Applications, pages 719-726. Springer, 2009.

[34] C. Masolo, G. Guizzardi, L. Vieu, E. Bottazzi, and R. Ferrario. Relational roles and qua-individuals. In AAAI Fall Symposium on Roles, an interdisciplinary perspective, pages 103-112, 2005.

[35] S. Murer, C. Worms, and F. J. Furrer. Managed evolution. Informatik-Spektrum, 31(6):537-547, 2008.

[36] C. Piechnick, S. Richly, S. Götz, C. Wilke, and U. Aßmann. Using Role-Based Composition to Support Unanticipated, Dynamic Adaptation - Smart Application Grids. In Proceedings of ADAPTIVE 2012, The Fourth International Conference on Adaptive and Self-adaptive Systems and Applications, pages 93-102, 2012.

[37] M. Pradel and M. Odersky. Scala roles: Reusable object collaborations in a library. In Software and Data Technologies, pages 23-36. Springer Berlin Heidelberg, 2009.

[38] T. Reenskaug and J. O. Coplien. The dci architecture: A new vision of object-oriented programming. An article starting a new blog:(14pp) http://www. artima. com/articles/dci_vision. html, 2009.

[39] J. Rumbaugh, R. Jacobson, and G. Booch. The Unified Modelling Language Reference Manual. Addison-Wesley, 1st edition, Jan. 1999.

[40] T. T. S. Monpratarnchai. The design and implementation of a role model based language, EpsilonJ. In Proceedings of the 5th International Conference on Electrical Engineering/Electronics, Computer, Telecommunications and Information Technology (ECTI-CON 2008), 2008.

[41] Y. Smaragdakis and D. S. Batory. Implementing layered designs with mixin layers. In Proceedings of the 12th European Conference on Object-Oriented Programming, ECCOP '98, pages 550-570, London, UK, UK, 1998. Springer-Verlag.

[42] F. Steimann. On the representation of roles in object-oriented and conceptual modelling. Data \&6 Knowledge Engineering, 35(1):83-106, 2000.

[43] G. Wielenga. On powerjava: "roles" instead of "objects". https://blogs .oracle.com/geertjan/ entry/on_powerjava_roles_instead_of, jan 2013. [Online; accessed 28-May-2014].

[44] H. Zhu and M. Zhou. Role-based collaboration and its kernel mechanisms. Systems, Man, and Cybernetics, Part C: Applications and Reviews, IEEE Transactions on, 36(4):578-589, July 2006. 